\title{
STRATEGIC ANALYSIS OF COMPETITIVENESS OF HIGH-TECH COMPANIES AS A TOOL FOR MANAGING THE REGION'S INNOVATIVE DEVELOPMENT
}

\author{
Yuriy A. Doroshenko, Mariia S. Starikova, Irina V. Somina, Irina O. Malykhina, Viktoriia N. Riapukhina* \\ Belgorod State Technological University named after V.G. Shukhov, Russian Federation
}

The sustainability of a high-tech business in a competitive environment which has been characterized by high turbulence is mainly due to its ability to adapt to changes that are already underway and anticipate future market transformations. Adaptability is a characteristic of an enterprise which gives by its management system. However, it depends on the state and degree of variability of the competitive environment. Despite the diversity of properties of the competitive environment, in our opinion, its complex characteristic that influences the ability of HTC to maintain stability to external changes taking place is turbulence, which has the property of mobility, complexity, uncertainty. Taking into account the analysis of the concept under study in physics and aerodynamics, we can consider turbulence to be irregular in time with the randomness of the fluctuations of the parameters of the competitive HTC environment. Our methodology for evaluating of CSHTC is based on the principles of complementarily and congruence and is based on measuring the discrepancy between the planned and achieved results on the indicators that are crucial for the key product groups of a high-tech company. Testing methods carried out on the example of high-tech companies in the pharmaceutical sector of the Central Federal District of the Russian Federation.

Key words: innovative development, environment, high-tech companies, competitiveness

\section{INTRODUCTION}

High-tech companies (HTC) are part of a market institution that provides strategic stability, security and innovative development of the region, therefore, analysis and strengthening of their competitive stability is a significant task both from the socio-economic side and from practical positions.

The sustainability of a high-tech business in a competitive environment which has been characterized by high turbulence is mainly due to its ability to adapt to changes that are already underway and anticipate future market transformations. K.N. Lee researched the influence of social and political problems on managing the sustainability of the development of socio-economic systems and pointed out the connection between the contradictions that arise in society and the instability of development(Lee, 1993). In the tasks of ensuring the competitive stability of entrepreneurial structures, adaptive management is considered as an approach to making management decisions that is applicable in conditions of high environmental uncertainty and at the same time with an objectively existing opportunity to influence it or its own positions in it (Williams et all,2009). The concept of adaptability of the enterprise was established in the 1970s, denoting the company's ability to adapt to changes in the external environment to ensure its viability (Child, 1972). The researches of organizational adaptability, developed in the 1960s, were based on the theory of circumstances (contingency theory) and studied the connection between an enterprise and its environment (Leavitt, 1965).An increase in the adaptability of high-tech companies to changes in the competitive environment leads to an increase in their sustainability, contributes to an increase in the capitalization of innovations (Schetininaet all, 2015), leads to an increase in interregional integration relations (Stolyarova et all, 2015). In this sense, adaptation becomes one of the processes of managing competitive stability, denoting actions to change the parameters of an economic entity in accordance with the results of monitoring, fixation of deviations in the dynamics of the development of the competitive environment and in the functioning of the HTC. In an economy based on knowledge, in which the importance of creative thinking and innovative approaches in activities is steadily increasing, emphasis is placed on the fact that adaptation is a learning-based process (Kremsater et all, 2003; Sturges,1926), therefore it is important to include in the sequence of steps in the competitive management process the resilience of actions for the formation of a data bank on the sensitivity of HTC to external changes. One of the important components of the management of HTC is a strategic analysis and assessment of competitive stability. At the same time, there is still a lack of clarity regarding the potencies of companies to stabilize their operations in an environment of uncertainty. The characteristic for living systems statement "the higher the uncertainty of the environment, the harder it is to predict the development of the situation and adapt to it" is true for the markets with the HTC.The author of chaos theory, E. Lorenz (Lorenz, 1993), proved the futility of attempts to predict the state of objects in chaotic systems, describing the "butterfly effect", according to which minor changes in the initial conditions give 
rise to huge differences in the final development. Indeed, as a result of globalization and the information technology revolution, the competitive environment at the end of the 20th century was changed very fast and its uncertainty only increased. Traditional methods of strategic analysis, developed for more predictable conditions of the external environment, have ceased to be successful. The emergence of adaptive "ecosystems" in business changes the notion of leadership, which is no longer necessarily based on the effect of experience and the effect of scale, which are traditional approaches applicable to a relatively stable environment. Therefore, part of the research on the problems of business sustainability in a competitive environment turned to the problem of intuitive planning (Courtney, 1997). On the one hand, it follows from this that managing competitive resilience of the HTC, based on forecasting, experimenting, learning, using knowledge about the sensitivity of an enterprise to environmental and environmental manifestations - to the effects of HTC, loses its relevance due to the spontaneity and uniqueness of changes in the business ecosystem. On the other hand, it is impossible to deny the cyclical nature of social, economic, political phenomena and the objective possibility of capturing market and macroeconomic trends, and, consequently, the assertion that the development of the business environment is chaotic is at least debatable. We have to say that some natural, climatic processes are difficult to predict and irreversible so the "butterfly effect" for forecasting and adaptive planning of ecological systems is more legitimate than for the formation of adaptive mechanisms of business structures whose environment is more predictable. But we have to note that the growth factor of the uncertainty of the external environment of enterprises and the resulting errors in the prediction of development cannot be ignored in managing the competitive stability of HTC.

\section{METHOD}

Adaptability is a characteristic of an enterprise which is givenby its management system. However, it depends on the state and degree of variability of the competitive environment. The characteristics of the competitive environment noted in various sources (Fredrickso\&laquinto, 1989; Miller\&Friesen, 1983; Nooraie, 2011; Papadakis et all, 1998) are: mobility (dynamism), speed of changes occurring in the external environment, complexity, uncertainty (degree of novelty of events), interconnectedness (heterogeneity) of factors; changes; turbulence. Despite the diversity of properties of the competitive environment, in our opinion, its complex characteristic that influences the ability of HTC to maintain stability to external changes, which has the property of mobility, complexity, uncertainty. Taking into account the analysis of the concept under study in physics and aerodynamics, we can consider turbulence to be irregular in time with the randomness of the fluctuations of the parameters of the competitive HTC environment.

The methodology which we offer in our research is based on the hypothesis that the competitive stability of the HTC determines by:

- the ability to adapt to external transformations, and therefore, the stability of functioning and the preservation of the dynamics of development in a competitive environment;

- competence of management, expressed, in particular, in anticipating the development of the situation in the external environment and in setting achievable, coordinated, realistic goals;

- the degree of turbulence of the HTC competitive environment, which can be used, in our opinion, as a corrective value: if the competitive environment was mobile during the estimated period, the lack of balanced development of HTC may be due not to the quality of management, but to environmental factors.

These arguments show necesserityto evaluate the two strategic components of the competitive sustainability of a high-tech company (CSHTC):

$$
\text { CSHTC }=\frac{\text { intrastability development a lof HTC }}{\text { turbulence of the competitive environment }}
$$

Our methodology for evaluating of CSHTC is based on the principles of complementarily and congruence and is based on measuring the discrepancy between the planned and achieved results on the indicators that are crucial for the key product groups of a high-tech company. Evaluation is made in the following steps:

1. Selection of main indicators of the development of HTC. We offer natural and value output and profit indicators, which can be considered as one of the key parameters of the region's innovative development.

2. Fixing the planned $(P)$ and actual values $(F)$ of the i-th general indicators for the j-th product areas. As a result, we formed matrices:

$$
\left(\begin{array}{ccc}
P_{11} & \ldots & P_{1 j} \\
\ldots & \ldots & \ldots \\
P_{i 1} & \ldots & P_{i j}
\end{array}\right) \quad\left(\begin{array}{ccc}
F_{11} & \ldots & F_{1 j} \\
\ldots & \ldots & \ldots \\
F_{i 1} & \ldots & F_{i j}
\end{array}\right)
$$

3. Measurement of the relative (percentage) deviation of actual values from the planned $\left(d_{i j}\right)$ :

$d_{i j}=\frac{F_{i j}-P_{i j}}{P_{i j}} \cdot 100$

The choice of relative rather than absolute deviations is due to the difference in units of measurement of general indicators.

As a result, we formed a matrix of relative deviations:

$\left(\begin{array}{ccc}d_{11} & \ldots & d_{1 j} \\ \ldots & \ldots & \ldots \\ d_{i 1} & \ldots & d_{i j}\end{array}\right)$

4. Evaluation of the intra-stability of the development of HTC units on the basis of averaging the relative deviations along the j-th product lines:

$$
\bar{d}_{j}=\sqrt{\frac{\sum_{i=1}^{n} d_{i j}^{2}}{n}}
$$


where $\mathrm{n}$ - is the number of main indicators of intra stability development.

The choice of standard deviations as a general indicator of development intra-stability is associated with the fact that $\mathrm{d}_{\mathrm{ij}}$ may have positive and negative values.

5. The calculation of the indicator of the intra-stability of the development of the HTC (B) based on the weighting of indicators characterizing the product areas $\left(\bar{d}_{j}\right)$ :

$$
\begin{aligned}
& B=\sum_{\mathrm{j}=1}^{\mathrm{m}} \bar{d}_{j} \cdot w_{j} \\
& w_{j}=\frac{V_{j}}{\sum_{j=1}^{m} V_{j}}
\end{aligned}
$$

where $w_{j}$ - significance factor (weight) of the relative developmental deviation, which demonstrates the degree of influence of the product line on corporate development, $\mathrm{j}=1, \ldots, \mathrm{m}$;

$$
\begin{aligned}
& V j \text { - revenue for the j-th product direction; } \\
& m \text { - number of product lines in HTC. }
\end{aligned}
$$

6. Evaluation of the intra-stability of the development of $\mathrm{HTC}$ in the dynamics of product areas $\left(B_{\text {dinamic }}\right)$ :

$B_{\text {dinamic }}=\frac{\sum_{t=1}^{k} B_{t}}{k}$

where $B_{t}-$ indicator of the stability of the corporation in the year $\mathrm{t}, \mathrm{t}=1, \ldots, \mathrm{k}$;

$k$ - the number of years in the study period.

This indicator is measured as a percentage since it represents the average difference over the entire HTC relative difference of the total actual estimation of performance indicators and their planned values.

7. Assessment of the variability of the competitive environment. The measurement of the mobility of this component of the environment is carried out in accordance with the following phases:

- selection of indicators on the principles of completeness of the situation in the relevant areas and representation in open sources of information;

- $\quad$ adding coefficients of significance $\left(w_{i} ; \sum w_{i}=1\right)$ to account for differences in the degree of influence for each factor of the competitive environment on the HTC. The relative importance of the components of the competitive environment can be obtained as a result of a survey of experts, who could be the leaders of the HTC of various industries;

- creationof a data bank for the assessment period of interest (medium-term interval);

- calculation of coefficients of variation of parameters $\left(\vartheta_{\text {compet } i}\right)$, which are describing each component of the competitive environment;

- determination of the standard value of the coefficient of variation $\left(\vartheta_{\text {norm }}\right)$,serving as a criterion for assessing the degree of turbulence of the competitive environment. The value of the coefficient of variation, which delimits the values indicating weak and strong turbulence, is selected as the normative coefficient of variation. Normally, it is considered that when the coefficient of variation is below $33 \%$, the considered population is considered to be homogeneous, that is, changing within acceptable limits characterizing the sustainability of development. However, this threshold value should be used for distributions close to normal. In our case, when analyzing the competitive HTC environment, the distribution is asymmetric, which requires a different approach to the choice of the standard value of the coefficient of variation. The choice of the standard value is carried out on the basis of constructing a cumulative curve of the coefficients of variation across the entire set of competitive environment indicators and fixing the value corresponding to the extremum of this curve.;

- determination of the weighted average ratio $\left(\vartheta_{\text {compet } i}\right)$ :

$v_{\text {compet }}=\sum_{i=1}^{n} v_{\text {compet }_{i}} \cdot w_{i}$

- comparison of the weighted average coefficient with the normative coefficient of variation gives reason to conclude on the degree of turbulence of the competitive environment during the study period: if $\vartheta_{\text {com- }}$ $>\vartheta_{\text {norm }}$, therefore, a competitive environment has high turbulence if $\vartheta_{\text {compet }}<\vartheta_{\text {norm }}$, therefore, the competitive environment has low turbulence. During 20062009. 13,08>11,93, in 2010-2013,12,57>12,3, which indicates a high turbulence of the macro-environment. At the same time, in the second time interval, its relative stabilization occurs;

- Assessment of the turbulence index of the competitive environment:

$I_{\text {turb }}^{\text {compet }}=\frac{v_{\text {compet }}}{v_{\text {norm }}}$

- If $I_{\text {turb }}^{\text {compet }}>1$, then the medium is considered highly turbulent, if $I_{\text {turb }}^{\text {compet }}<1$, then the medium is considered low turbulent. Converting the coefficient of variation, measured as a percentage, into an index view will allow it to be used to adjust the intra-stability index of the HTC.

8. Evaluation of CSHTC using the indicator CS:

CSHTC is measured in percent. Converting it into an index view seems to be unreasonable, since its economic meaning is connected with the demonstration of the degree of deviation from the planned development of the HTC. The expediency of using the turbulence indicator of the competitive environment in the index form is due to the fact that it is in this case a correction value. The scaling of the values of the CS indicator to determine the assessment of the CSHTC is carried out on the basis of table 1. 
Table 1 - Scale of CSHTC levels, \% variation (formed on the basis of a survey of experts)

\begin{tabular}{|c|c|l|c|c|}
\hline $\begin{array}{l}\text { Normal, } \\
\text { natural }\end{array}$ & $\begin{array}{l}\text { Acceptable, } \\
\text { maintainable } \\
\text { controllable } \\
\text { HTC }\end{array}$ & $\begin{array}{l}\text { Problem, indicating a lack of } \\
\text { competitive stability of the } \\
\text { HTC }\end{array}$ & $\begin{array}{l}\text { Critical, due to } \\
\text { significant gaps between } \\
\text { the internal and external } \\
\text { environment of the HTC }\end{array}$ & Invalid \\
\hline 7 & 15 & 22 & 31 & 40 \\
\hline
\end{tabular}

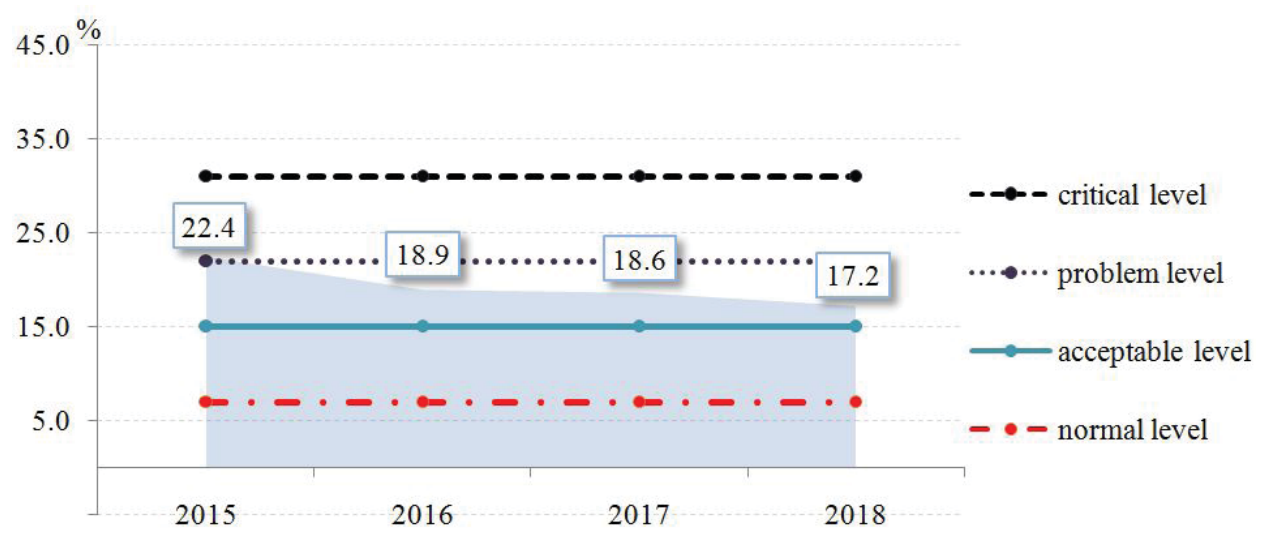

Figure 1: CS Level of LLC "PIK-PHARMA",\% variation

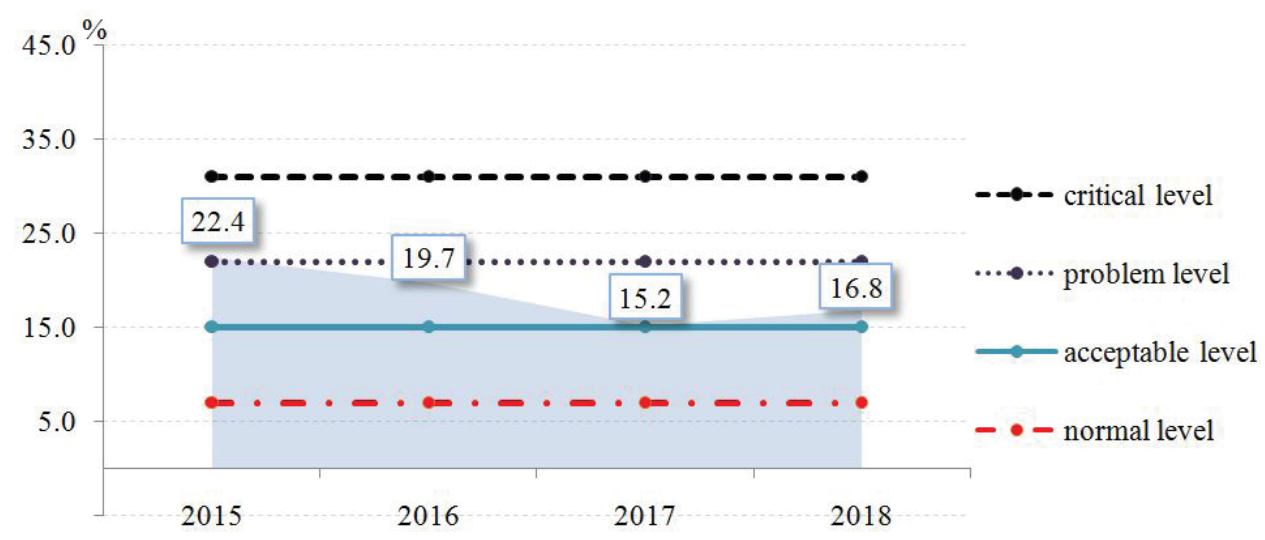

Figure 2: CS Level of JSC "R-PHARM", \% variation

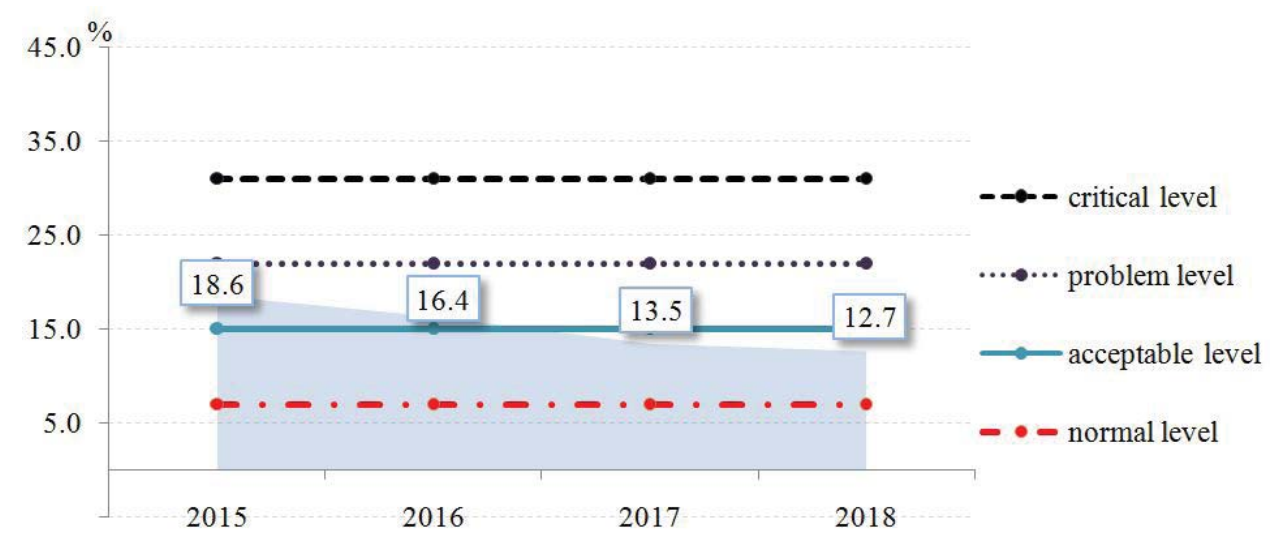

Figure 3: CS Level of PJSC "Pharmstandard",\% variation 


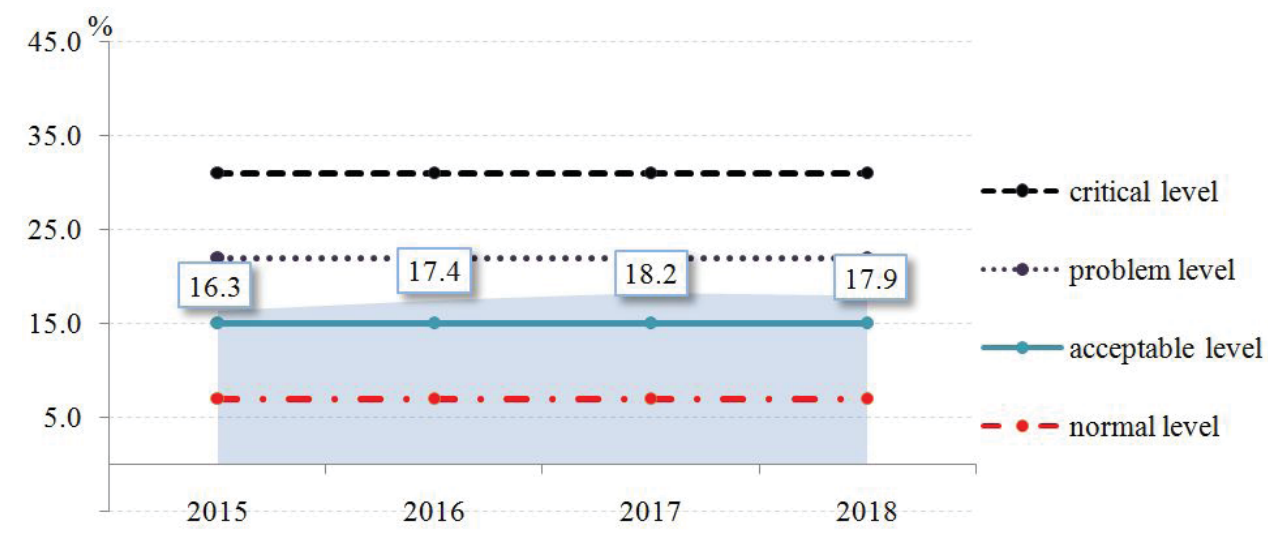

Figure 4: CS Level of CJSC "Implementation Center "Protek"', \% variation

\section{Competitive Environment}

Turbulence Index

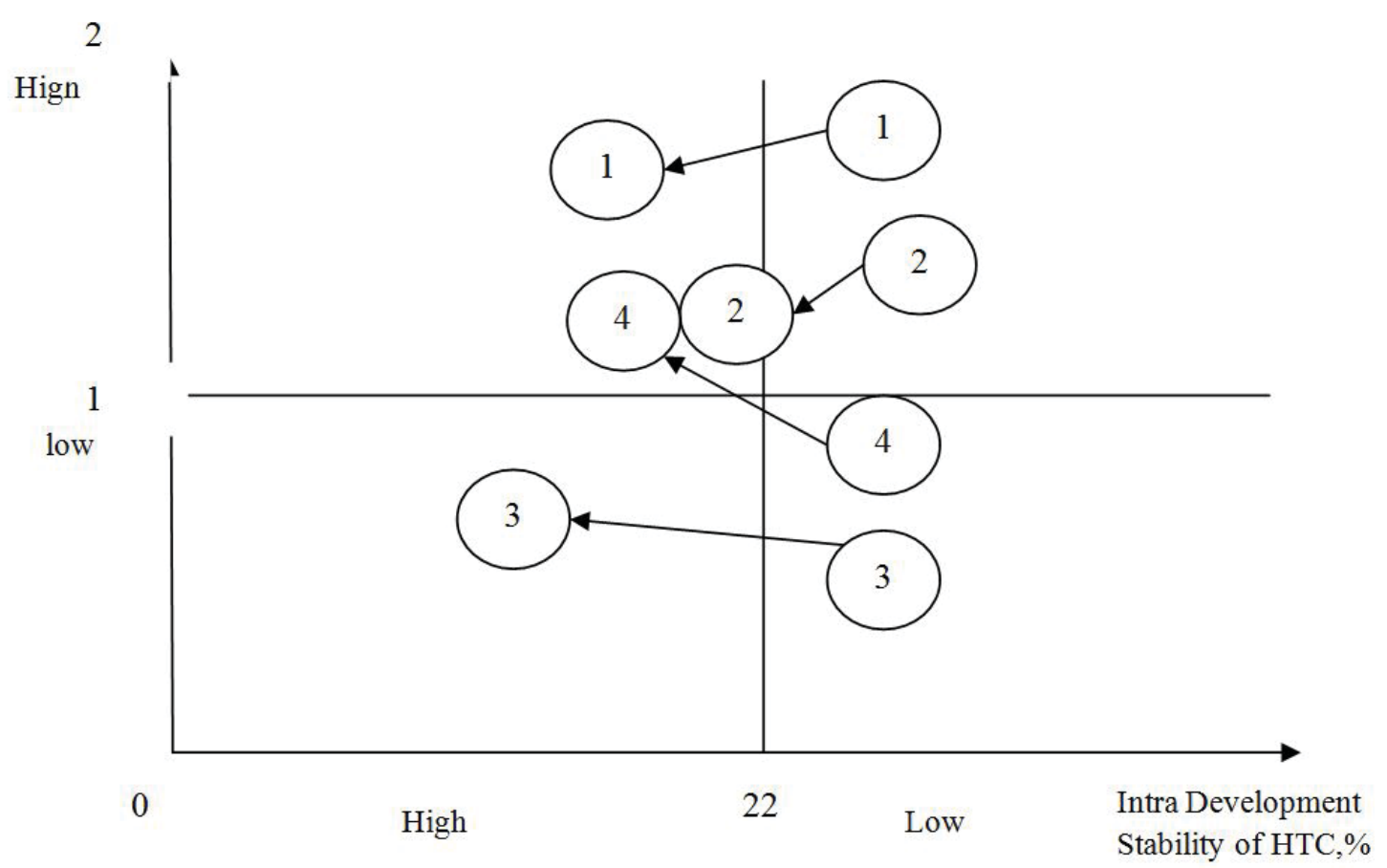

Figure 5: Matrix of the strategic analysis of competitive stability of pharmaceutical companies according to sales data on target market segments in 2015 and 2018,\% variation

\section{RESULTS}

Testing methods carried out on the example of high-tech companies in the pharmaceutical sector of the Central Federal District of the Russian Federation. The evaluation results are shown in fig.1-4.

\section{Discussion of the results}

During the period which we review high-tech companies in the pharmaceutical sector in general increased their competitive stability, changed the problem level of CS to an acceptable level. The calculations used a single indicator of the turbulence index of the competitive environment, recorded during each year within the national pharmaceutical market. At the same time, it is possible to get estimates for market segments, which will allow to more clearly determine the competitive position of HTC. And in this case, the turbulence index will be different in terms of the segments and the companies operating on them, which allows to build a matrix (Fig. 5), allowing to evaluate the effectiveness of management actions in relation to the strategic cores of the business. It is clearly seen that in the case of companies No. 1, 2, in the face of a decrease in the turbulence of the competitive environment, there is an increase in the sustainability of the company. Companies No. 3, 4 are characterized by an increase in the competitive stability of basic activities in a situation of increasing uncertainty in the competitive environment, which indirectly indicates a higher synergistic efficiency of the HTC data. 


\section{CONCLUSIONS}

1. Competitive stability of a high-tech company in a market environment is due both to the effectiveness of management decisions and the turbulence of the external environment, which is interpreted as the time-irregular randomness of fluctuations of its parameters. Since the existing models for assessing the variability of the HTC environment, have drawbacks (they use mainly qualitative measurement methods, are difficult to verify, fragmentary, etc.), the article proposed and tested a method for assessing the competitive stability of the HTC, based on measuring the turbulence of the competitive environment and the intra stability of the HTC development.

2. Testing the methodology showed an objective opportunity to made conclusions regarding the prospects for changes in the product portfolio and the effectiveness of management actions in the field of ensuring the competitive stability of the HTC at the corporate level and at the level of individual strategic business units.

\section{ACKNOWLEDGEMENTS}

The work was prepared during implementation of project No 26.9642.2017/8.9 within the framework of the State task of the Ministry of Education and Science of Russia.

\section{REFERENCES}

1. Lee, K.N. (1993). Compass and Gyroscope - Integrating Science and Politics for the Environment: Washington, D.C., Island Press, 243.

2. Williams, B.K., Szaro,R.C. and Shapiro, C.D. (2009). Adaptive management: Washington, US Department of the Interior, 74.

3. Child, J. (1972). Organizational structure, environment and performance: The role of strategic choice. Sociology, 6 (1), 1-22.

4. Leavitt, H. (1965). Applied Organizational Change in Industry: Chicago, Rand McNally, 359.

5. Schetinina, E.D., Doroshenko, Y.A., Ovtcharova, N.V. (2015). The problems of innovations commercialization and approaches to their decision: International Business Management, 9, 6, 1074-1078.

6. Stolyarova, V.A., Kupriyanov, S.V., Stolyarova, Z.V., Saldanha, H.F.D., Sukhraj, R.A. (2015). The role of science as a factor of integration of countries in the context of globalization:Asian Social Science, 11, 7, 278-283.

7. Kremsater, L.F., Bunnell,D.H. \&Dunsworth,G. (2003). Indicators to assess biological diversity: Weyerhaeuser's Forest Project,The Forestry Chronicle, 79, 590-601.

8. Sturges, H. (1926). The choice of a class-interval: J. Amer. Statist. Assoc., 21, 65-66.
9. Lorenz, E. (1993). Essence of chaos: UCL Press, 240.

10. Courtney, H.G., Kirkland,J. \&Viguerie,S. (1997). Strategy in an uncertain world: Harvard Business Review, 5, 81-90.

11. Fredrickson, J.W. \&laquinto,A.L. (1989). Inertia and creeping rationality in strategic decision processes: Academy of Management Journal, 32, 543-576.

12. Miller, D. \& Friesen,P.H. (1983). Strategy Making and Environment: The Third Link. Strategic Management Journal, 4, 221-235.

13. Nooraie, M. (2011). Decision's familiarity and strategic decision-making process output: the mediating impact of rationality of the decision-making process: International Journal of Applied Decision Sciences (IJADS), 4, 385-400.

14. Papadakis, V.M., Lioukas,S. \&Chambers,D. (1998). Strategic decision-making processes: the role of management and context: Strategic Management Journal, 19, 115-147.
Paper submitted: 27.06.2019.

Paper accepted: 25.11.2019.

This is an open access article distributed under the CC BY-NC-ND 4.0 terms and conditions. 